\title{
Good Design, The Secret of Successful Complete Denture Prostheses
}

\author{
University of Edinburgh Emeritus Professor
}

David M. Watt

Good design is the secret of success in complete denture prostheses but some of the techniques, that are commonly used prevent the construction of welldesigned dentures.

It is generally accepted that complete denture prostheses should represent the lost tissues in the same amounts and in the same positions from which they were lost. This presents few difficulties when immediate dentures are made, but, with each successive denture it becomes more and more difficult to determine the size and shape of the lost tissues and thus the design of successional dentures deteriorates with time until it is difficult to make satisfactory dentures when extreme resorption has taken place.

A 17-year longitudinal study of oral changes following the loss of teeth has provided a clear description of "The space in the mouth that was formerly occupied by the teeth and their supporting structures that have since been lost". I feel that this is a better definition of the "denture space" than those found in current glossaries of dental terms, as knowledge of it has led to a radical revision of our techniques for designing and making dentures to fill that space (Fig. 1).

The measurements that were made to construct the average diagrams in Figure 1 showed a remarkable constancy of the bucco-lingual breadth of the alveolar ridge measured horizontally from the lingual gingival margins to the buccal mucosa midway between the buccal sulcus and the level of the lingual gingival margin, before the teeth were ex-

11 Lockharton Garden Edinburgh EH14 1AU.

Scotland, UK. tracted (Fig. 1). The constancy of this measurement at each tooth position was so striking in the original sample that we measured altogether 100 patients to check that the variation was really as small as we observed. The results of those measurements are shown in Table 1 . Note that the standard deviation is only about $1 \mathrm{~mm}$ at each tooth position so that the average measurement plus or minus about $1 \mathrm{~mm}$ will be correct for about $68 \%$ of patients and plus or minus about $2 \mathrm{~mm}$ for about $95 \%$ of the patients. You can check this by measuring casts in your laboratory. Thus if we can locate the position of the lingual gingival margins in the edentulous mouth, we have a reasonable guide for repositioning the lips and cheeks correctly, because they lie against the buccal surfaces of the dentulous ridges.

The remnant of the lingual gingival margins is represented in the edentulous mouth by a fibrous cord that crosses the ends of the rugae as it follows the line of the residual ridge (Fig. 2). It lies near the crest of the ridge when there has been little resorption but where resorption is extreme it lies near the vestibular sulcus. Difficulties in treating patients with marked resorption arise because a small impression tray is selected to fit the shrunken ridge, and, as it does not restore the full breadth of the sulcus, the impression material dribbles over its edge and consequently the sulcus on the cast is too narrow and it is then impossible to design the denture correctly. A denture made on a small cast will not be retentive as it is rather like a loose piston in the soft cylinder of the mouth-it can easily be 

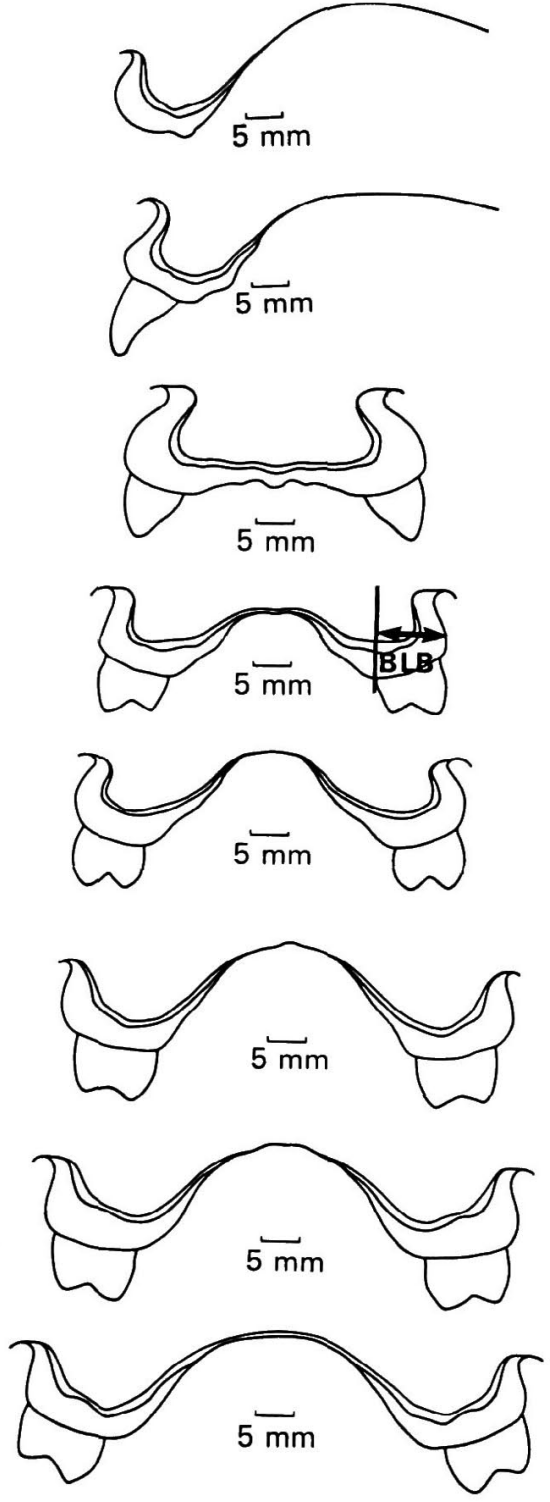

Fig. 1 Average changes in the mixillary denture-bearing area $2 \frac{1}{2}$ and $14-17$ years after extraction of the teeth. The pre-extraction bucco-lingual breadth of the alveolar process in the first premolar region is marked BLB

withdrawn. An adequately extended denture is like a well-fitting piston, firmly retained (Fig. 3).

\section{Biometric Impression Technique}

It is the function of the impression tray to hold the

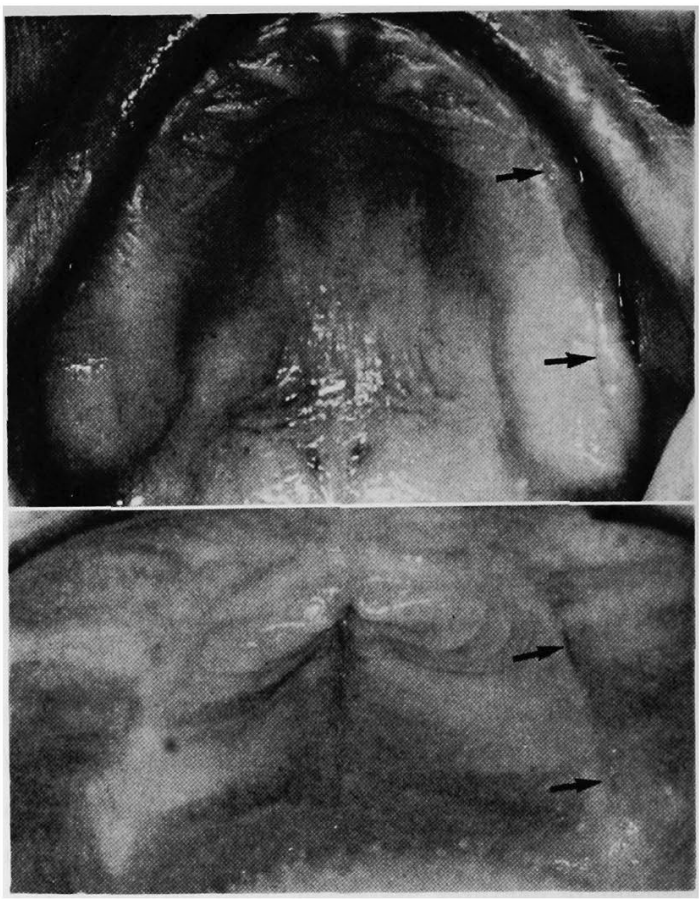

Fig. 2 Top: The remnant of the lingual gingival margins (arrows) on a maxillary ridge showing little resorption, lies near the crest of the ridge

Bottom : On a ridge showing marked resorption, the remnant of the lingual gingival margins (arrows) lies near the vestibular sulcus. In this case the "ridge" comprises the soft compressible tissue at the sides of the palate

lips and cheeks in their pre-extraction positions while the fluid impression material simply records the form of the vestibular sulcus appropriate to the breadth recorded by the tray. Thus biometric trays are constructed so that that their buccal surfaces hold the lips and cheeks at the appropriate average distances from the remnant of the maxillary lingual gingival margins. As it is unlikely that you would remember the figures in table 1 , the following are sufficiently near for most clinical purposes :-

Incisor $\quad 6 \mathrm{~mm}$
Canine $\quad 8 \mathrm{~mm}$

Premolar $10 \mathrm{~mm}$

Molar $12 \mathrm{~mm}$

To provide a cast on which a biometric tray can be constructed, the stock tray for the preliminary impression should not be selected to fit the residual ridge but should hold the cheeks and lips at these 

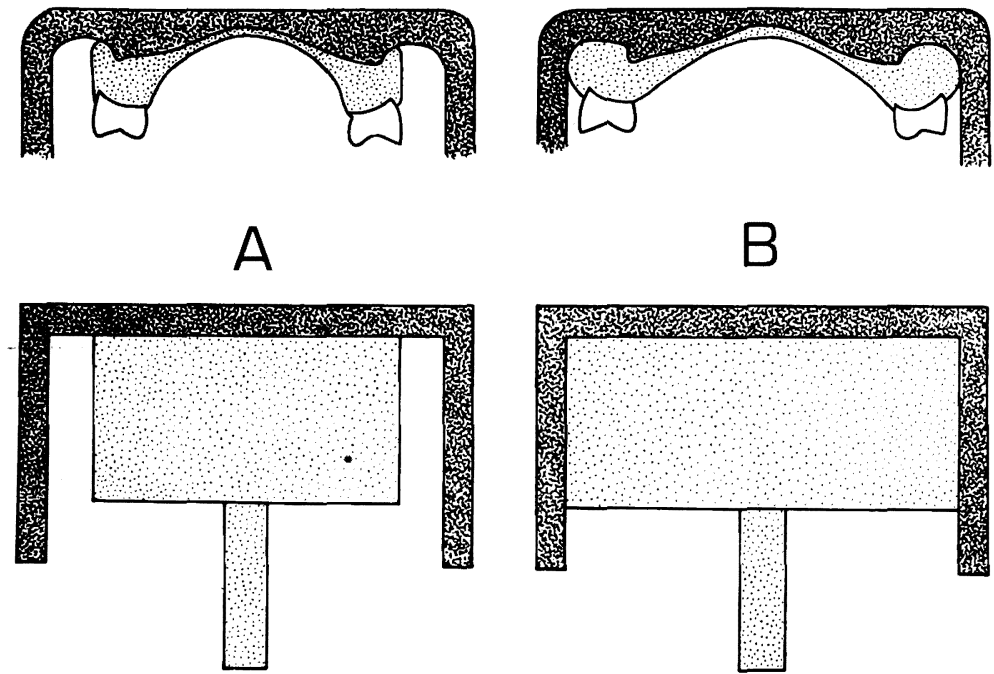

Fig. 3 A. A denture made on a small cast will not be retentive as it is rather like a loose piston in the soft cylinder of the mouth

B. An adequately extended denture is like a well fitting piston, firmly retained

average distances from the lingual gingival remnant. Thus when the ridge is large the tray flange will be thin but when the ridge is small the flange of the biometric tray will be thick (Fig. 4). In this way a buccal valve seal is developed so that when a post. dam of tracing stick is placed across the biometric tray, before the impression is taken, the empty tray is retentive, and needless to say the impression is even more retentive even on the flattest maxillary ridge.

When the upper denture is designed to restore all the lost maxillary tissue an additional advantage accrues. The denture periphery holds the cheek out and increases the space available for the lower denture. When unsupported, the inward collapse of the lips and cheeks have an adverse affection the lower impression especially if the lower ridge is flat. If a little flat tray is used the cheeks, lip and tongue flop over it and the peripheral form of the impression has a cross-section like a $U$ lying on its side. Thus when the impression is cast, most of the sulcus must be cut away in order to remove the impression from the cast. Biometric lower trays are designed to prevent lip tongue and cheeks collapsing over the tray and to hold them in their former upright positions so that the impression ma-
Table 1 Analysis of measurements of horizontal breadths of alvolar processes from lingual gingival margins to mid-buccal points in 100 dentulous patients

\begin{tabular}{lcc}
\hline \hline $\begin{array}{c}\text { Position and plane } \\
\text { of measurement }\end{array}$ & $\begin{array}{c}\text { Average } \\
\text { Measurement } \\
(\mathrm{mm})\end{array}$ & $\begin{array}{c}\text { Standard } \\
\text { Deviation } \\
(\mathrm{mm})\end{array}$ \\
\hline Central incisor ( sagittal) & 6.3 & 0.91 \\
Canine (coronal) & 8.5 & 1.06 \\
First premolar (coronal) & 10.0 & 1.03 \\
Second premolar (coronal) & 10.6 & 1.40 \\
First molar (coronal) & 12.8 & 0.98 \\
Second molar (coronal) & 11.6 & 1.14 \\
Third molar (coronal) & 10.1 & 1.33 \\
\hline
\end{tabular}

terial is supported as it runs round the edge of the tray and up between the tray and the tissues. In this way the full contour of the sulcus is restored (Fig. 5). A ridge runs along the back of the tray to support the peripheral tissues. The cross-section form of the ridge in incisor, premolar and molar regions is shown in the lower part of the figure. The rounded buccal flange in the molar region ensures that the contour of the buccal pouch is properly delineated. As the middle fibres of the buccinator contract they "bow-string" across from the fixed corner of the mouth to the pterygo-mandibular raphe at the level of the occlusal plane and would not 


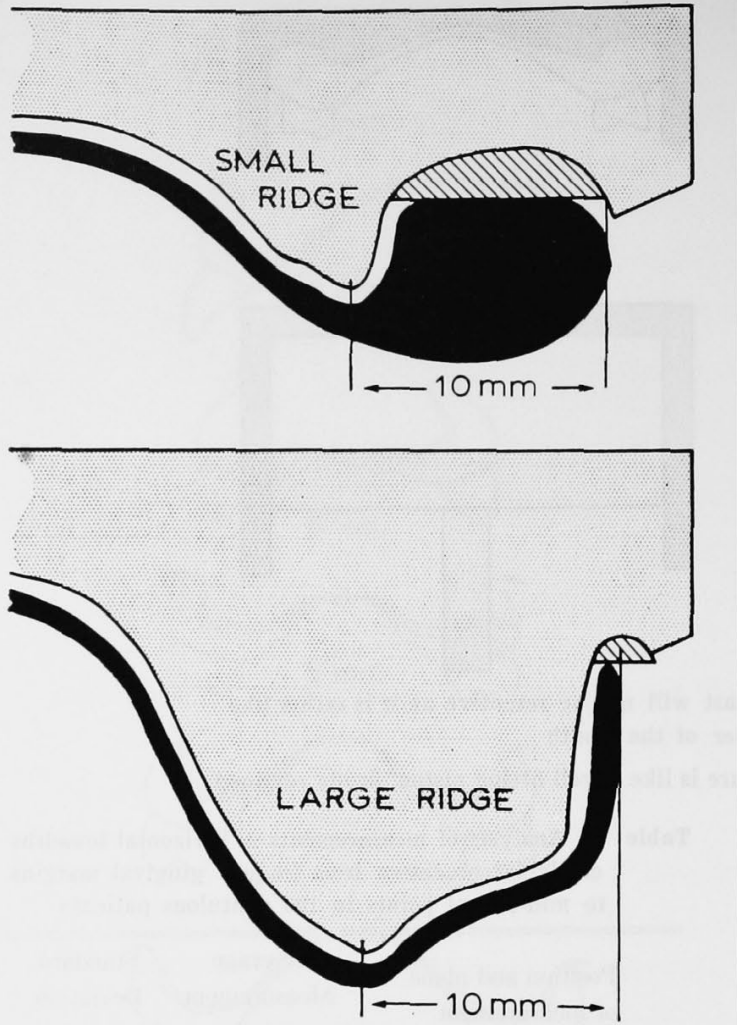

Fig. 4 Coronal sections through maxillary casts and trays in the left premolar region. Where there has been much bone loss the residual ridge is small and the buccal flange of the biometric tray is thick. Where the ridge is large the tray flange is correspondingly thinner. In each case the measurement from the remnant of the lingual gingival margins to the buccal surface of the tray is $10 \mathrm{~mm}$. This represents the average bucco-lingual breadth in the premolar region of the dentulous maxilla

contact a concave buccal surface but do rest against a convex surface and help to stabilise the denture.

The shaded area in Figure 6 represents a coronal section of a lower denture in the left molar region. It is superimposed on a coronal section of the mandible with the natural molar tooth present. Note that the bucco-lingual breadth of the artificial molar tooth is little more than the bucco-lingual breadth of the buccal cusp of the natural molar. This provides a sloping lingual surface for the tongue to rest on and stabilise the lower denture. Note that the convex buccal flange goes beyond the denture
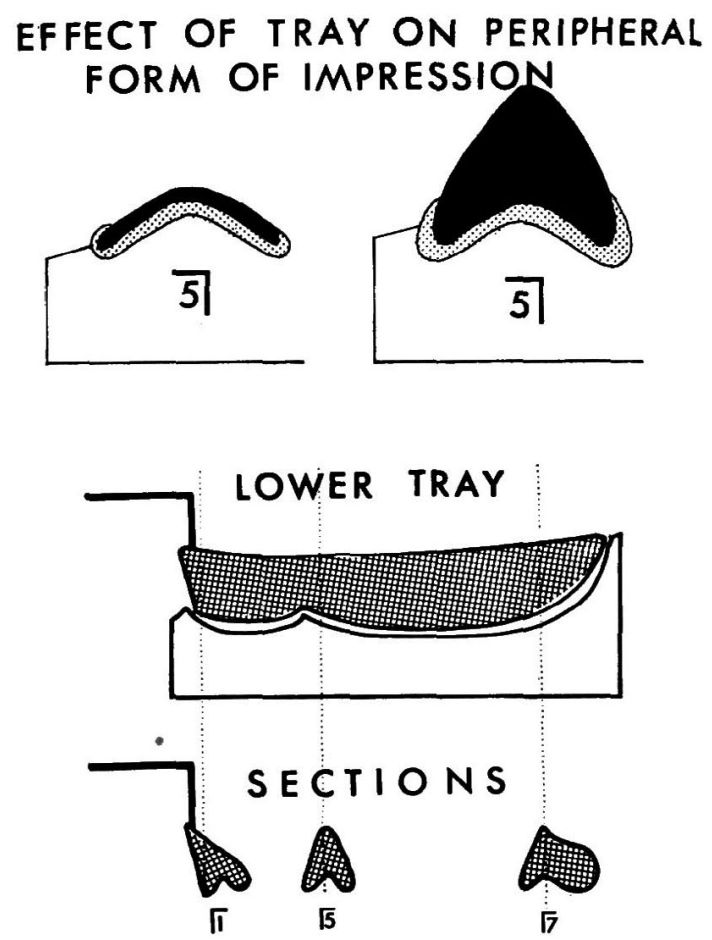

Fig. 5 Top left : cross section of second right premolar region of a conventional impression tray for a flat lower ridge. The impression material flops over the top of the tray and the peripheral form is abnormal

Top right: Cross section of a biometric tray that supports cheek and tongue so that the impression material gives a more accurate representation of the form of the buccal and lingual sulci

Lower diagram : Represents a lower biometric tray showingcross sections in incisor, premolar and molar regions

space as I have defined it, in order to fill the buccal pouch and maintain contact with the buccinator and prevent food collecting in the pouch.

There should be an area of denture base about $1 \mathrm{~cm}$ long distal to the last molar to provide a "posterior shelf " upon which the tongue can rest on the lingual side and the cheek on the buccal side to stabilise the lower denture.

When the mouth is open the tongue is withdrawn to guard the oropharynx and it takes a conscious effort on the part of the patient to place his tongue in the functional position with its tip on the lingual surfaces of the upper anterior teeth and its dorsum 


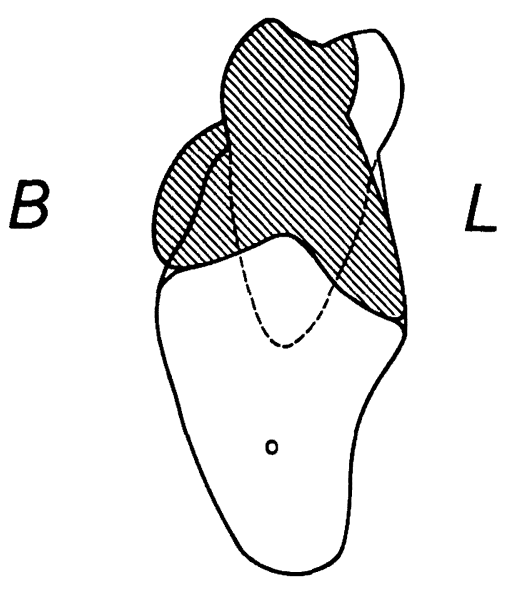

Fig. 6 Coronal section of the left molar region. $B$, buccal $L$, lingual

The shaded area represents the section through the prosthesis superimposed on a section of the mandible with the natural tooth present. Note that the bucco-lingual breadth of the artificial molar is little more than the buccolingual breadth of the buccal cusp of the natural molar. This provides a sloping lingual surface for the tongue to rest on and stabilise the lower denture. Note that the convex buccal flange of the lower denture goes beyond the denture space as I have defined it, in order to maintain contact with the cheek and prevent food collecting in the buccal pouch

against the palate. When taking lower impressions the tongue should be forward and up in the functional position and should not be moved from side to side but should be kept quite still. In this position the floor of the mouth is flat but in the guarding position the floor of the mouth is lowered and the lingual pouch becomes much deeper. You can easily tell if there is a lingual overextension of the impression by looking at where the groove formed by the mylohyoid ridge crosses the lingual margin of the impression. If it crosses in front of the second molar region, the impression is overextended and the denture is likely to rise when the tongue is slightly protruded.

\section{Jaw Relationships}

Now let us consider the changes in denture po-
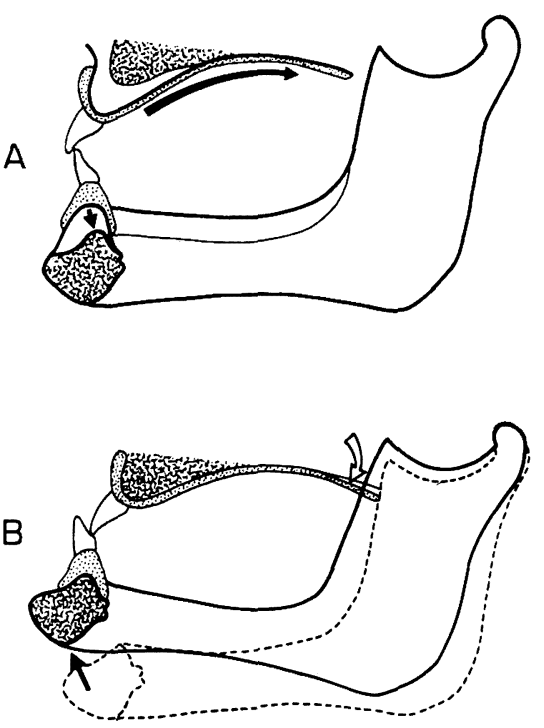

C

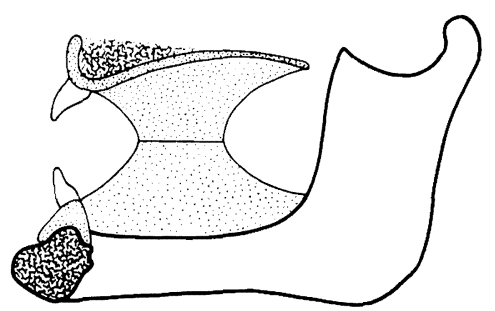

Fig. 7 Diagrams of the changes in denture position and jaw relationship that occur after prolonged wearing of complete dentures

A. The position of the dentures shortly after fitting. The shaded areas represent the shape of the ridges after 20 years of marked resorption

B. Twenty years later the dentures are now seated on the resorbed ridges

C. Occlusal pivots restore the mandible to its pre-extraction position (See text for further details)

sition and jaw relationship that occur on prolonged wearing of complete dentures. Large resorptive changes in the edentulous jaws produce large changes in the position of the mandible and hence large changes in the occlusion of the complete dentures (Fig. 7).

Diagram A Figure 7 shows the position of the complete dentures in the mouth shortly after fitting. The shaded areas represent the shape of the ridges after twenty years of marked resorption. The arrows 

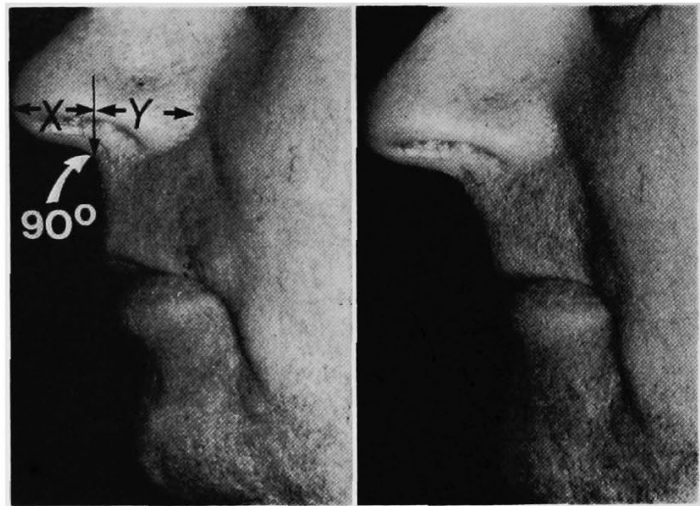

Fig. 8 Right: When the teeth are lost the dental bulge disappears and the lips fall in

Left: Guides to the restoration of the dental bulge. The centre of the curved junction between columella and philtrum is about half way between the tip of the nose and groove behind the ala. $\mathrm{X}=\mathrm{Y}$. The angle between the columella of the nose and the philtrum of the lip is approximately $90^{\circ}$ but many factors affect it (See Fig. 9)

show the movement of the dentures as they maintain contact with the resorbing ridges.

Diagram B. The dentures, twenty years later, are now seated on the resorbed ridges. Forward movement of the mandible accompanies its elevation which is sufficient to compensate for the resorption of both maxillary and mandibular ridges. The forward movement brings the mandibular incisors into labial relationship with the maxillary ones. The upper denture slides backwards round the curve of the palate so that it tends to lose contact at the post-dam area (open arrow) but thickening of the palatal mucosa may compensate for this. During these changes the vertical ramus of the mandible acts as a prop and protects the ridges posteriorly. Thus the occlusal force is greatest anteriorly and most resorption takes place there.

Diagram C. Restoration of the mandible to its pre-extraction position by means of occlusal pivots, produces a wide incisor gap because the changes in the supporting structures have radically altered the position of the dentures in the mouth. The difficulties of repositioning the dentures by additions to their fitting surfaces are such that it is better to
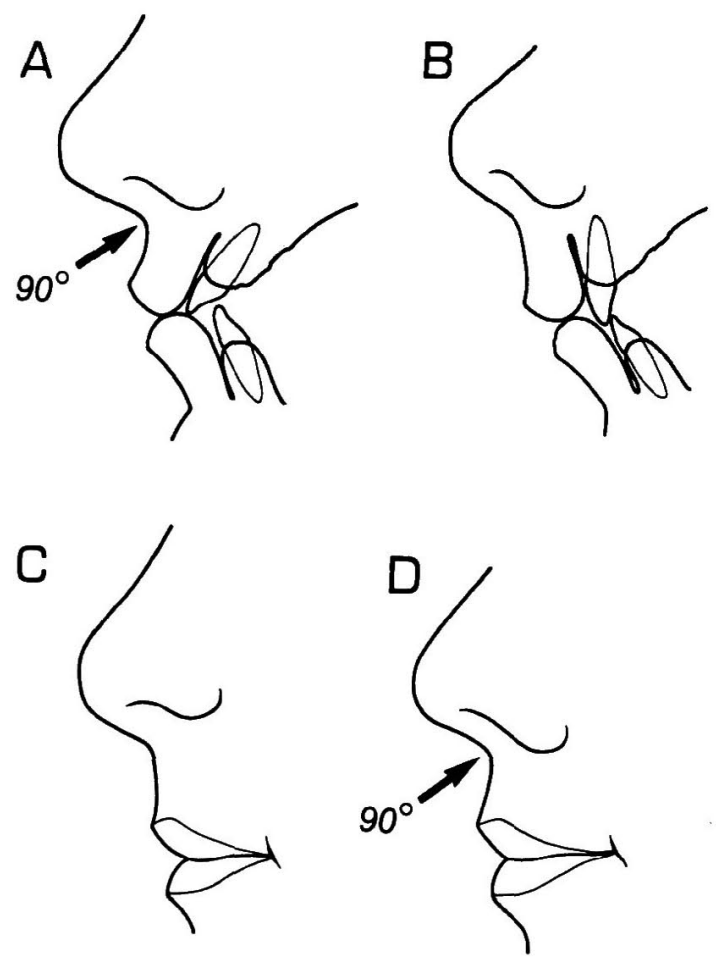

Fig. 9 The effect of tooth inclination and nose form on the naso-labial angle

A. When the teeth are proclined, the naso-labial angle is about $90^{\circ}$

B. When the teeth are retroclined the naso-labial angle may be more than $90^{\circ}$

C. When the columella is prominent and the ala high the angle is more than $90^{\circ}$

D. When the columella and ala are at the same level, the naso-labial angle is about $90^{\circ}$

establish the jaw relationship with occlusal pivots then remake the dentures on casts from impressions taken inside the dentures after suitable tissue conditioning. It must be remembered that many of the difficulties that we may experience in the registration of jaw relationships can be avoided by placing pivots in the second premolar region of the old dentures and allowing time for the adaptive changes that have taken place in the joints, muscles and jaws, over many years to reverse and establish a stable jaw relationship.

I would recommend that all dentures, more than a few years old, that have to be replaced, should have clear acrylic pivots placed and adjusted until the 


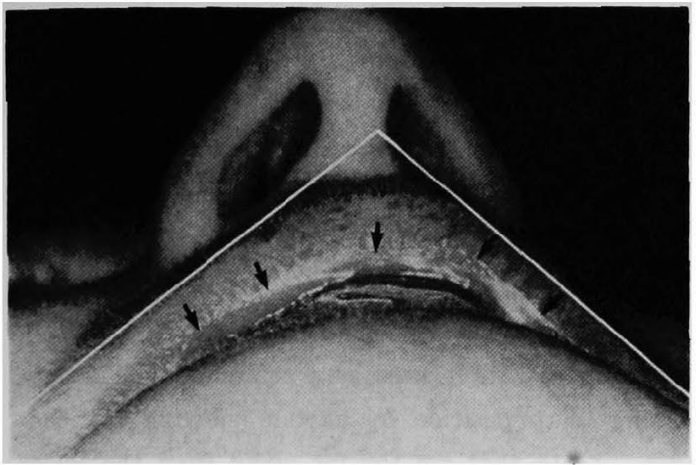

Fig. 10 The horizontal labial angle varies between $90^{\circ}$ and $120^{\circ}$

When the lip is correctly supported, about $3 \mathrm{~mm}$ of glossy oral mucosa can be seen between the record block and the transitional epithelium of the lip. The junction between the two epithelia (arrowed) can be clearly seen in this photograph

occlusion is stable before any attempt is made to finally register the jaw relationship. Much trouble can be avoided by this procedure.

The stability of the occlusion is checked with a stereostethoscope or an occlusal sound recorder to ensure that successive contact sounds are of short duration and similar wave form. This means that the patient can find the same occlusal position time after time and thus has established an "occlusal engram" in the brain that represents base line for all occlusal activity.

\section{The Appearance of Complete Dentures}

When a sculptor is modelling a face he takes a piece of clay about the size of a golfball and sticks it below the nose in order to model the lips. This is the dental bulge which is present when the natural anterior teeth are present but, when they are lost, the dental bulge disappears (Fig. 8). The buccal strap, comprising the buccinators and orbicularis oris, which surrounds the facial curtain can be drawn tight, and in the absence of the anterior teeth, distorts the facial curtain and severely reduces the size of the denture space. For this reason functional movements of lips and cheeks during impression taking should be discouraged as it will be impossible
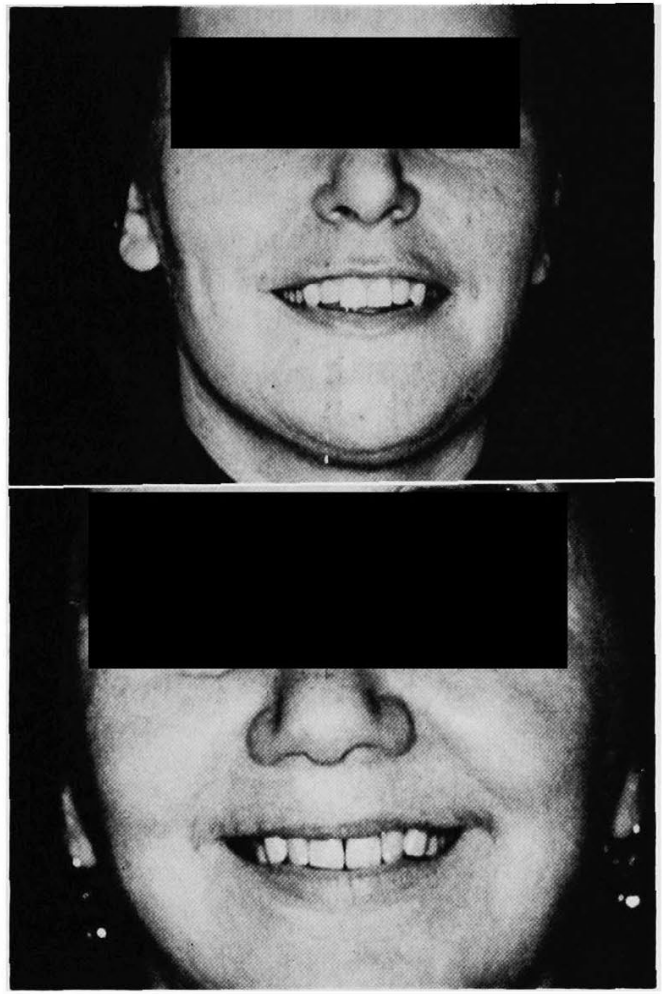

Fig. 11 Top: The appearance of this complete upper denture is marred by the positions of the canine teeth which look too long because they do not follow the curve of the smiling lower lip

Bottom: This complete denture looks better because the teeth have been set to follow the curve of the smiling lower lip

to construct a well-designed denture on the cast made from a functional impression as its periphery will be too narrow. The majority of complete artificial dentures can be detected without the patient even opening her mouth simply because the dental bulge is not properly restored. In the first instance the restoration must be done at the impression stage so that the cast will have a sufficiently broad vestibular sulcus to enable the anterior teeth to be placed in the positions occupied by their natural predecessors. Gujdes to the restoration of the dental bulge are as follows:-

1. The middle of the curved junction between the columella of the nose and the philtrum of the upper lip lies approximately half way between 
the tip of the nose and the groove behind the ala. $X=Y$ (Fig. 8) When the nose is long $X>Y$ but when it is short $\mathrm{X}<\mathrm{Y}$.

2. The angle between the columella and the philtrum is $90^{\circ}$ in the classical profile but the inclination of the teeth and the prominence of the columella affect the size of this angle (Fig. 9).

3. The horizontal labial angle between the right and left sides of the upper lip varies between $90^{\circ}$ and $120^{\circ}$. Narrow faced individuals have the lower angle and the angle tends to be greater in broad-faced people (Fig. 10).

4. When the upper lip is correctly supported you should see about $3 \mathrm{~mm}$ of glossy oral mucosa between the record block (or labial surfaces of the incisors) and the transitional epithelium of the upper lip (Fig. 10).

There is always a tendancy to underfill the upper lip because when the lower lip is unsupported a correctly supported upper lip looks too prominent. A further guide to anterior tooth position is that the labial surfaces of the central incisors should lie about $10 \mathrm{~mm}$ anterior to the middle of the incisive papilla.
This measurement is seldom less than eight or more than twelve millimetres.

Anterior teeth on complete dentures are usually too small because they are set too far back on too narrow an arc which cannot accommodate teeth of the correct size.

When setting upper anterior teeth make sure that their incisal edges follow the curve of the smiling lower lip as this is used as a reference line for assessing tooth position by anyone viewing the denture. Teeth that are too far away from the lower lip are assessed as being too short and teeth that overlap it as too long. A very common fault is to set the incisors on a flat piane. This immediately shows them to be artificial (Fig. 11).

\section{Note}

No references have been included in this summary as a complete list of references can be found in “Designing Complete Dentures" 2nd Edition. David M. Watt \& A. Roy MacGregor. Wright, Bristol, 1986. 